\title{
Remdesivir in Treatment of COVID-19: A Systematic Benefit-Risk Assessment
}

\author{
Miranda Davies $^{1,2}$ (1) Vicki Osborne ${ }^{1,2} \cdot$ Samantha Lane $e^{1,2} \cdot$ Debabrata Roy $^{1,2} \cdot$ Sandeep Dhanda ${ }^{1,2} \cdot$ Alison Evans $^{1,2}$. \\ Saad Shakir ${ }^{1,2}$
}

Published online: 28 May 2020

(c) The Author(s) 2020

\begin{abstract}
Introduction There is a need to identify effective, safe treatments for COVID-19 (coronavirus disease) rapidly, given the current, ongoing pandemic. A systematic benefit-risk assessment was designed and conducted to examine the benefit-risk profile of remdesivir in COVID-19 patients compared with standard of care, placebo or other treatments. A key objective of this study was to provide a platform for a dynamic systematic benefit-risk evaluation, which starts with inevitably limited information (to meet the urgent unmet public health need worldwide), then update the benefit-risk evaluation as more data become available.

Methods The Benefit-Risk Action Team (BRAT) framework was used to assess the overall benefit-risk of the use of remdesivir as a treatment for COVID-19 compared with standard of care, placebo or other treatments. We searched PubMed, Google Scholar and government agency websites to identify literature reporting clinical outcomes in patients taking remdesivir for COVID-19. A value tree was constructed and key benefits and risks were ranked by two clinicians in order of considered importance.

Results Using the BRAT method, several key benefits and risks for use of remdesivir in COVID-19 compared with placebo have been identified. In one trial, the benefit of time to clinical improvement was not statistically significant ( 21 vs 23 days, HR $1.23,95 \%$ CI $0.87-1.75$ ), although the study was underpowered. In another trial, a shorter time to recovery in patients treated with remdesivir was observed (11 vs 15 days), with non-significant reduced mortality risk (8\% vs $12 \%)$. Risk data were only available from one trial. This trial reported fewer serious adverse events in patients taking remdesivir (18\%) compared with the placebo group (26\%); however, more patients in the remdesivir group discontinued treatment as a result of an adverse event compared with those patients receiving placebo (12\% vs 5\%).

Conclusions Preliminary clinical trial results suggest that there may be a favourable benefit-risk profile for remdesivir compared with placebo in severe COVID-19 infection and further data on benefits would strengthen this evaluation. There is limited safety data for remdesivir, which should be obtained in further studies. The current framework summarises the key anticipated benefits and risks for which further data are needed. Ongoing clinical trial data can be incorporated into the framework when available to provide an updated benefit-risk assessment.
\end{abstract}

\section{Introduction}

Coronaviruses are a cause of respiratory tract infections in humans [1]. A novel coronavirus emerged in Wuhan, China in December 2019 [2], subsequently called Severe Acute

Miranda Davies

miranda.davies@dsru.org

1 Drug Safety Research Unit, Bursledon Hall, Blundell Lane, Southampton SO31 1AA, UK

2 School of Pharmacy and Biomedical Sciences, University of Portsmouth, Portsmouth, UK
Respiratory Syndrome coronavirus 2 (SARS-CoV-2) [3]. Coronavirus disease (COVID-19) is caused by SARS-CoV-2 [3] and since March 2020 this outbreak has been declared a pandemic by the World Health Organisation (WHO) [4]. Fever, cough and shortness of breath are the main reported symptoms of COVID-19 [5] but it also has a concerning case mortality rate among certain populations, such as older adults and those with underlying health conditions.

Currently, there is a need to identify effective, safe treatments for COVID-19 as quickly as possible, due to the ongoing pandemic. One such proposed treatment is remdesivir, which is an investigational antiviral medicine with proven activity against RNA viruses [6, 7]. Remdesivir was 


\section{Key Points}

COVID-19 is a global pandemic, for which remdesivir has been proposed as a possible treatment.

Preliminary clinical trial results suggest that there may be a favourable benefit-risk profile for remdesivir compared with placebo; however, data is limited at the current time.

Ongoing clinical trial data can be incorporated into the framework when available to provide an updated benefit-risk assessment.

originally developed for treatment of Ebola virus disease and results from the phase III randomised clinical trial for this indication have been published [8]. It has been shown to have broad spectrum in-vitro activity against several coronaviruses, including SARS-CoV-2 [6, 9, 10], in-vivo activity against MERS-CoV in animal models [11, 12], and has been made available for patients with COVID-19 through compassionate use programmes [13,14]. Several clinical trials are ongoing to examine the effectiveness and safety of remdesivir in COVID-19 in humans [15-18]. Most recently, remdesivir was granted an Emergency Use Authorisation in COVID-19 by the US Food and Drug Administration [19]. Whilst there is considerable interest in the use of remdesivir for COVID-19, there has not been a systematic benefit-risk assessment on the use of remdesivir for COVID-19 treatment using a structured descriptive framework. A key objective of this study was to provide a platform for a dynamic systematic benefit-risk evaluation, which starts with inevitably limited information (to meet the urgent unmet public health need worldwide), then update the benefit-risk evaluation as more data become available.

The ongoing monitoring of the benefit-risk balance for remdesivir in COVID-19 treatment is strengthened by use of a systematic assessment. The Benefit-Risk Action Team (BRAT) framework facilitates identification and summarisation of the key benefits and risks of a product in a defined disease context within a structured descriptive framework; further quantitative assessments can then be applied and conducted according to the availability of relevant data at that time [20]. The BRAT framework was also specifically designed to assist communication with regulatory authorities [21, 22]. The decision-making process is transparent due to the framework design, while any assumptions can be explored further by sensitivity analysis through a quantitative component [20,23].

This benefit-risk assessment has been conducted based on publicly available publications to date (data-lock April
$30,2020)$. It is, however, acknowledged that there is extremely limited data available at this timepoint. The benefit-risk assessment has been designed to be implemented regardless of the quantity of data available, but the intention is that the framework will subsequently be readily available to repeat the assessment as further data arise (e.g. results from new and ongoing clinical trials), allowing for rapid decision making.

\section{Objectives}

The objective of this study was to examine the benefit-risk profile of remdesivir in COVID-19 patients compared with standard of care, placebo or other treatments.

\section{Methods}

\subsection{Benefit-Risk Framework}

The BRAT framework was used to assess the overall benefit-risk of using remdesivir as a treatment for COVID-19 compared with standard of care, placebo or other treatments. BRAT uses a six-step iterative process to support the decision and communication of a benefit-risk assessment: define decision context, identify outcomes, identify data sources, customise framework, assess outcome importance and display and interpret key benefit-risk metrics [21]. We identified three settings of interest for use of COVID-19 treatments; treatment for severe disease, treatment of milder disease in the community, and prevention in healthcare professionals exposed to the virus. We have focused on the use of remdesivir for the treatment of severe COVID-19 disease within this benefit-risk assessment. For the purposes of this study, we considered severe COVID-19 to include any patient who was hospitalised as a result of the infection.

\subsubsection{Population of Interest}

The population of interest were patients with severe COVID19 , while the exposure of interest was remdesivir. The comparators of interest were standard of care, placebo or other treatments for COVID-19.

\subsubsection{Outcomes of Interest}

Regardless of importance, all potential benefits and risks related to remdesivir were initially identified. Key benefits and risks were then selected by clinician judgement, which were those considered to drive the benefit-risk balance of remdesivir. The key benefits and risks were used to construct a value tree, ranking benefits and risks in order of considered 
importance. Ranking was performed by two clinicians after discussion of the importance of each benefit and risk.

\subsubsection{Data Sources and Customisation of the Framework}

We searched PubMed, Google Scholar and government agency websites to identify suitable data for inclusion. The search strategy for PubMed and Google Scholar was.

Remdesivir AND (coronavirus OR covid* OR "SARSCoV-2" OR "2019-NCov").

For government agency websites, we searched for any mention of 'remdesivir' on the National Institute of Health (NIH), US Food and Drug Administration and European Medicines Agency websites. Papers were included if they reported quantitative data on effectiveness and/or safety of remdesivir and a comparator in patients with severe COVID19. Case reports were excluded because they did not provide comparative data. Results were restricted to English language only (abstracts in English language were acceptable where sufficient data was provided) and peer-reviewed publications (for PubMed and Google Scholar) since 2019 to 30 April, 2020. Where a control group was included, data were extracted for each benefit and risk, for both remdesivir and the comparator (standard of care, placebo or other treatments).

We also searched ClinicalTrials.gov to identify background information for the manuscript and to identify ongoing clinical trials. Since there are no results currently published on ClinicalTrials.gov, there were no useable data available for extraction.

\subsection{Outcome Assessment}

A summary benefit-risk table was created to allow visualisation of the magnitude of each benefit and risk. Risk differences and corresponding 95\% confidence intervals (CI) were calculated for each outcome where both numerator (number of events) and denominator (number of patients at risk) were available for both the treatment group (remdesivir) and comparator group.

\subsubsection{Quantitative Assessment}

Due to paucity of data, a fully quantitative assessment was not undertaken. However, the risks and risk difference per 1000 patients were calculated for each benefit and risk. The outcomes identified in the value tree were ranked so that swing weighting can be applied in future assessments. The weighted net clinical benefit ( $w N C B$ ) can subsequently be calculated using these weights, if sufficient data on both risks and benefits become available in the future [23-25]. We would propose using the Sutton et al. method, where benefits have a positive contribution to the $\mathrm{WNCB}$ and risks have a negative contribution [25]; the overall wNCB would be considered positive (benefit outweighs the risk) where wNCB is $>0$. A sensitivity analysis can also be used to examine the robustness of the assigned weights and whether significant changes would alter the benefit-risk profile [22]. Analysis using wNCB was not undertaken at this time due to the limited release of clinical trial data.

\section{Results}

Figure 1 displays the value tree of the key benefits and risks related to remdesivir treatment in COVID-19. The benefits included in the value tree include key endpoints from clinical trial protocols for studies assessing the efficacy of remdesivir in severe COVID-19 disease. As remdesivir is currently not approved for use in any condition, it is acknowledged that its safety profile has not been completely characterised. For the purposes of identifying potential risks associated with the use of remdesivir in COVID-19 disease for the value tree, safety data has been identified from currently available sources that met the inclusion/exclusion criteria. These include studies reporting its use in the treatment of Ebola virus disease [8], case series documenting the use of remdesivir in COVID-19 disease [26], and safety data included in the study by Wang et al. [27]. Both the efficacy and safety outcomes have been presented in ranked numerical order according to perceived clinical significance. The key characteristics of clinical trials for remdesivir in any population have been presented in Table 1. As per the BRAT method, only studies that met the inclusion/exclusion criteria for the current disease context (severe COVID-19) were included in the data extraction phase.

\subsection{Benefits}

The benefits listed in the value tree represent key clinical endpoints included in clinical trial protocols. These have been ranked in order of perceived clinical importance, from the clinical endpoint of mortality risk through to the surrogate endpoint of viral clearance. Many studies have utilised ordinal scales, which include a spectrum of the clinical status of the patient. The primary endpoint used in the recently published study by Wang et al. [27] was time to clinical improvement up to Day 28; this was defined as the time (in days) from randomisation to the point of a decline of two levels on a six-point ordinal scale of clinical status (from $1=$ discharged to $6=$ death) or discharged alive from hospital, whichever came first. The six-point scale was as follows: death $=6$; hospital admission for extracorporeal membrane oxygenation or mechanical ventilation $=5$; hospital admission for non-invasive ventilation or high-flow 


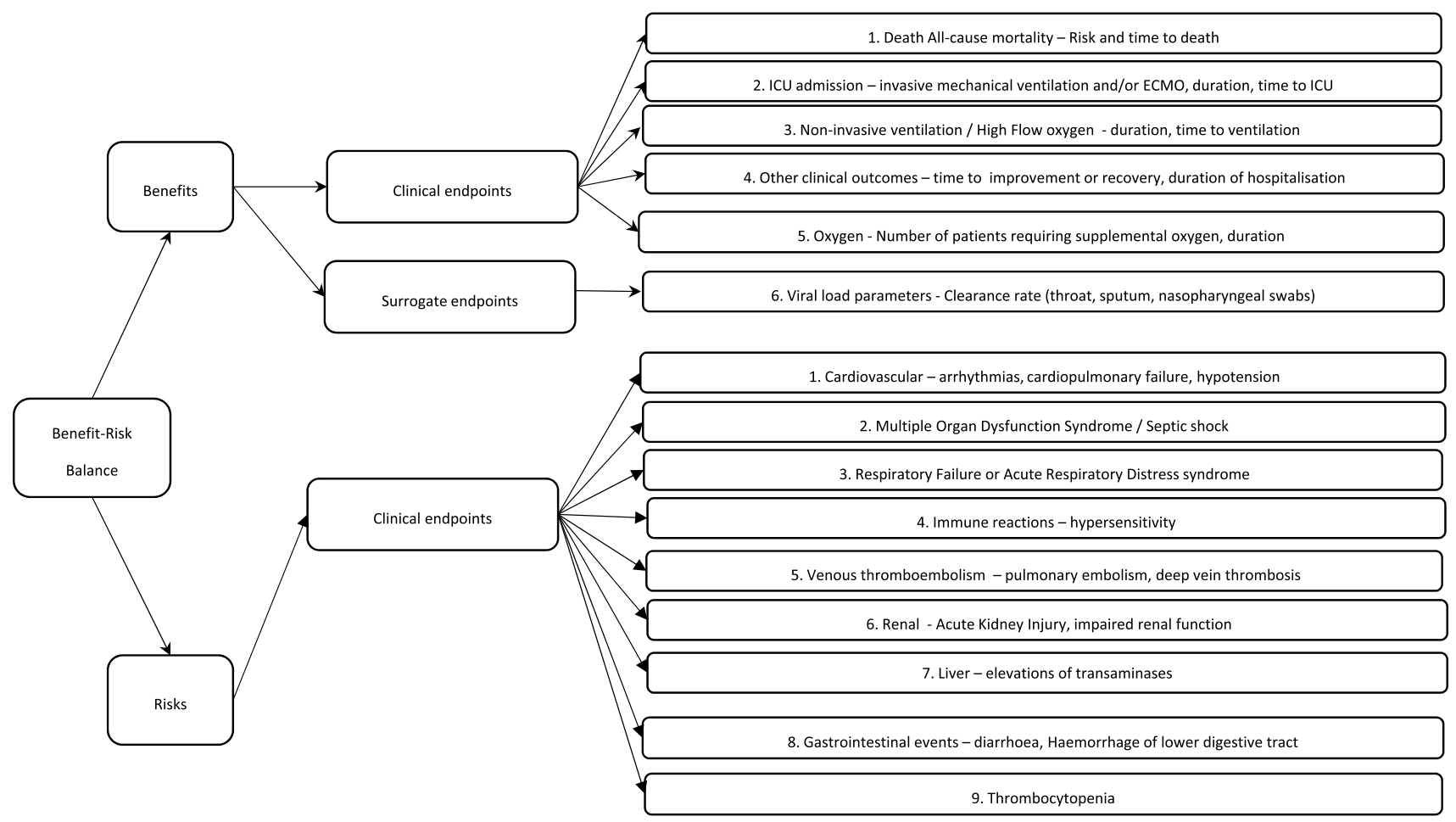

Fig. 1 Value tree of key benefits and risks identified for remdesivir, ranked by order of clinical significance

Table 1 Key characteristics of cited clinical trials for remdesivir

\begin{tabular}{lllll}
\hline Study & Disease context & Primary outcome & Relevant benefits examined & Relevant risks examined \\
\hline $\begin{array}{l}\text { Mulangu et al. [8] } \\
\text { Wang et al. [27] }\end{array}$ & $\begin{array}{l}\text { Ebola virus disease } \\
\text { Severe COVID-19 }\end{array}$ & $\begin{array}{l}\text { Death at 28 days } \\
\text { Time to improvement }\end{array}$ & $\begin{array}{l}\text { N/A } \\
\text { Death by Day 28, invasive ventilation, } \\
\text { non-invasive ventilation, oxygen } \\
\text { support, time to clinical improve- } \\
\text { ment, viral load }\end{array}$ & $\begin{array}{l}\text { Hypotension, elevated transaminases } \\
\text { tachycardia, multiple organ dysfunc- } \\
\text { tion syndrome, septic shock, acute } \\
\text { respiratory distress syndrome, hyper- } \\
\text { sensitivity, pulmonary embolism, } \\
\text { deep vein thrombosis, acute kidney } \\
\text { injury, elevated ALT, lower gastroin- } \\
\text { testinal haemorrhage, thrombocyto- } \\
\text { penia, adverse events, serious adverse } \\
\text { events }\end{array}$ \\
& & & Not published at this time
\end{tabular}

oxygen therapy $=4$; hospital admission for oxygen therapy (but not requiring high-flow or non-invasive ventilation) $=3$; hospital admission but not requiring oxygen therapy $=2$; and discharged or having reached discharge criteria $=1$.

The primary endpoint in the Multicentre, Adaptive, Randomized Blinded Controlled Trial of the Safety and Efficacy of Investigational Therapeutics for the Treatment of COVID19 in Hospitalized Adults, known as the Adaptive COVID19 treatment trial sponsored by the National Institute of Allergy and Infectious Diseases (NIAID) [28], was time to recovery (time frame: Day 1 through Day 29). Day of recovery was defined as the first day on which the subject satisfied one of the following three categories from the ordinal scale: (1) hospitalized, not requiring supplemental oxygen-no longer requires ongoing medical care; (2) not hospitalized, limitation on activities and/or requiring home oxygen; (3) not hospitalized, no limitations on activities. Other key clinical outcomes included duration of ventilation, duration of oxygen support, and duration of hospital stay, in addition to time from randomization to discharge or death. Surrogate endpoints assessing viral load clearance are likely to be less robust forms of endpoint data, and accordingly have been ranked lower in terms of clinical importance. 


\subsection{Risks}

As remdesivir is an unapproved medicine in most countries, its safety profile has not yet been fully characterised. Known key risks at the current time have been included in the value tree, identified from a variety of sources, including recently published clinical trial data in the context of COVID-19 [27]. These have also been ranked according to perceived seriousness.

Cardiovascular outcomes including hypotension and arrhythmias have been documented following the use of remdesivir [8, 26, 29]; however, the risk of cardiovascular outcomes with remdesivir remains largely unknown [30]. Hypotension was reported in one patient in the phase III study investigating remdesivir in the context of Ebola virus disease; this patient subsequently suffered a cardiac arrest, although the manuscript states that this death could not readily be distinguishable from underlying fulminant Ebola virus disease itself [8]. Cardiac arrest was also reported in a further patient in the remdesivir group reported in the COVID19 study by Wang et al. [27]. Multiple organ dysfunction, septic shock, acute kidney injury and hypotension have also been reported as adverse events amongst patients provided with remdesivir either on a compassionate-use basis, or in a clinical trial [26, 27]. Respiratory failure or acute respiratory distress syndrome has been cited as an adverse event in patients taking remdesivir [27, 31], and as such has been included here, although it is acknowledged that this may be related to underlying disease (COVID-19), rather than to remdesivir. Elevations in liver transaminases and gastrointestinal events, including diarrhoea, have also been reported with the use of remdesivir $[8,13,26,32]$, in addition to reports of haemorrhage of the lower gastrointestinal (GI) tract $[27,33]$.

\subsection{Quantitative Data}

Where available, clinical trial data relating to any efficacy or safety outcomes included in the value tree for both remdesivir and a comparator group in the context of severe COVID19 were extracted and included in Table 2. Based on this data, summary metrics including risks per 1000 patient years and risk differences are presented in Table 3. We identified 68 papers through literature searching from PubMed and 384 papers from Google Scholar. We also identified results from one clinical trial on the NIAID website. After initial review, one paper [27] and results from one clinical trial on the NIAID website [28] were included in the final benefit-risk assessment.

In the Wang et al. trial, the benefit of time to clinical improvement was not statistically significant (21 vs 23 days, HR $1.23,95 \%$ CI $0.87-1.75$ ). In the NIAID trial, a statistically significant shorter time to recovery in patients treated with remdesivir was observed (11 vs 15 days; $p<0.001)$, with non-significant reduced mortality risk ( $8 \%$ vs $12 \%$; $p=0.059)$. Other non-significant benefit data were identified from the Wang et al. trial, including invasive ventilation and oxygen use at Day 28 (1\% vs $4 \%$ and $12 \%$ vs $17 \%$, respectively).

Risk data were only available from the Wang et al. trial, which reported fewer serious adverse events in patients taking remdesivir (18\%) compared with the placebo group (26\%); however, more patients in the remdesivir group discontinued treatment as a result of an adverse event compared with those patients receiving placebo ( $12 \%$ vs $5 \%)$.

A serious adverse event is any event that results in death, is life-threatening, requires hospitalisation or prolongation of existing hospitalisation, results in persistent or significant disability or incapacity, or is a birth defect.

\section{Discussion}

This benefit-risk assessment presented the currently known key benefits and risks for the use of remdesivir for severe COVID-19 disease. The value tree provides a visual summary of these key benefits and risks, which have been ranked according to perceived clinical importance. As remdesivir is currently unlicensed in most countries, these include endpoints used in remdesivir clinical trials, and risks identified from available data sources. A literature search (including government agency websites) identified relevant numerical data for these outcomes for both remdesivir and comparator groups. Recently, the first results from a randomised, doubleblind, placebo-controlled clinical trial studying remdesivir in the context of COVID-19 have been published [27]. The primary outcome suggested a reduction in the median time to clinical improvement, although this difference was nonsignificant (21 vs 23 days, HR 1.23, 95\% CI 0.87-1.75). However, the planned sample size of 453 patients (151 on placebo and 302 on remdesivir) was not reached due to difficulties in enrolment, and therefore this study may have been underpowered to detect significant differences. Nevertheless, multiple additional endpoints were included in this study, and these data have been presented in Table 1. Whilst no statistically significant differences were observed between the remdesivir and placebo group [27], the study reported generally improved outcomes in the remdesivir group compared with placebo, including reductions in the risk of invasive and non-invasive ventilation, and need for supportive oxygen at day 28 .

Data has also been presented for remdesivir and the placebo group included in the Adaptive COVID-19 treatment trial [28]. As of 29 April, 2020, limited data has been made available on the NIH website [28] and suggested a statistically significant reduction in time to recovery from 


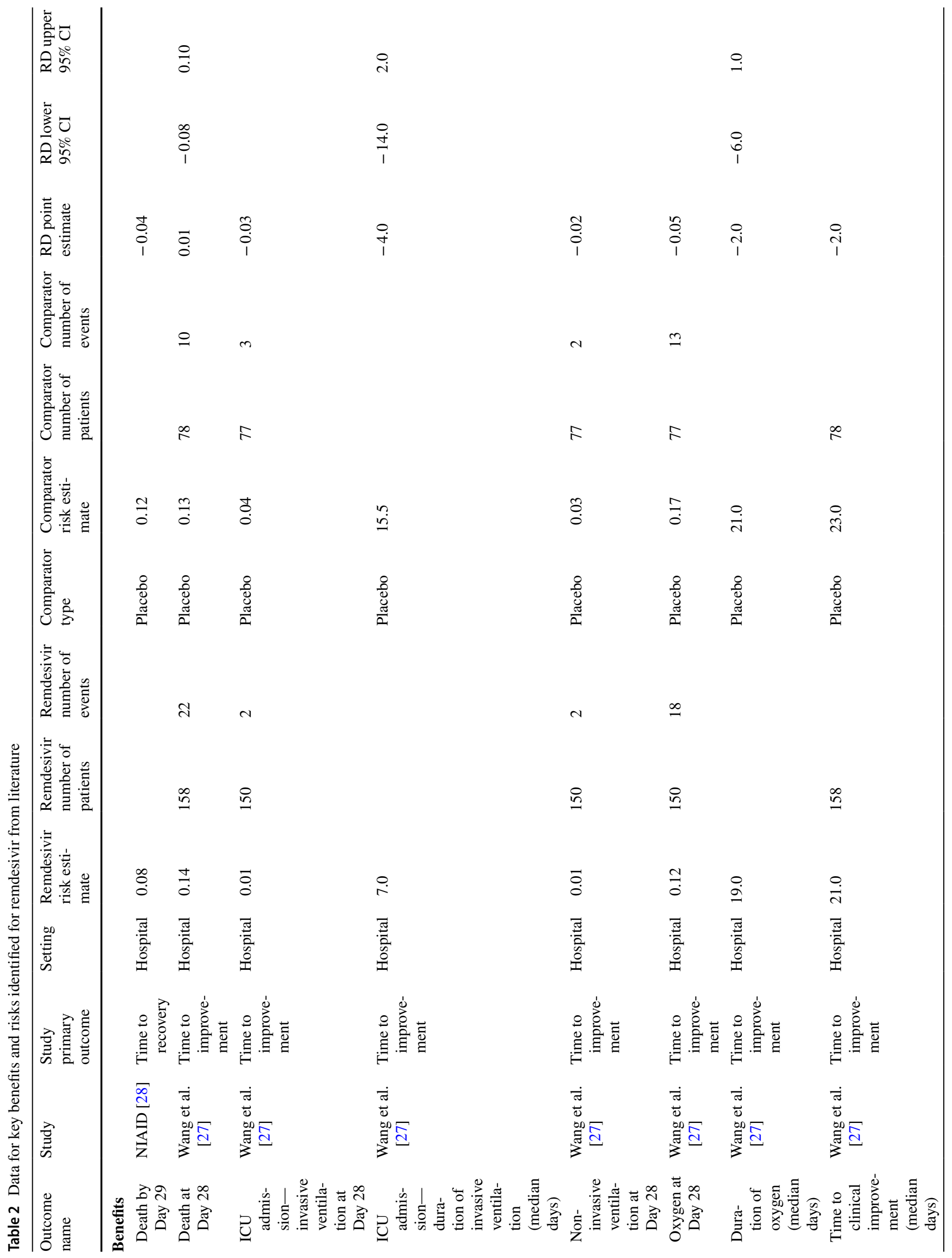




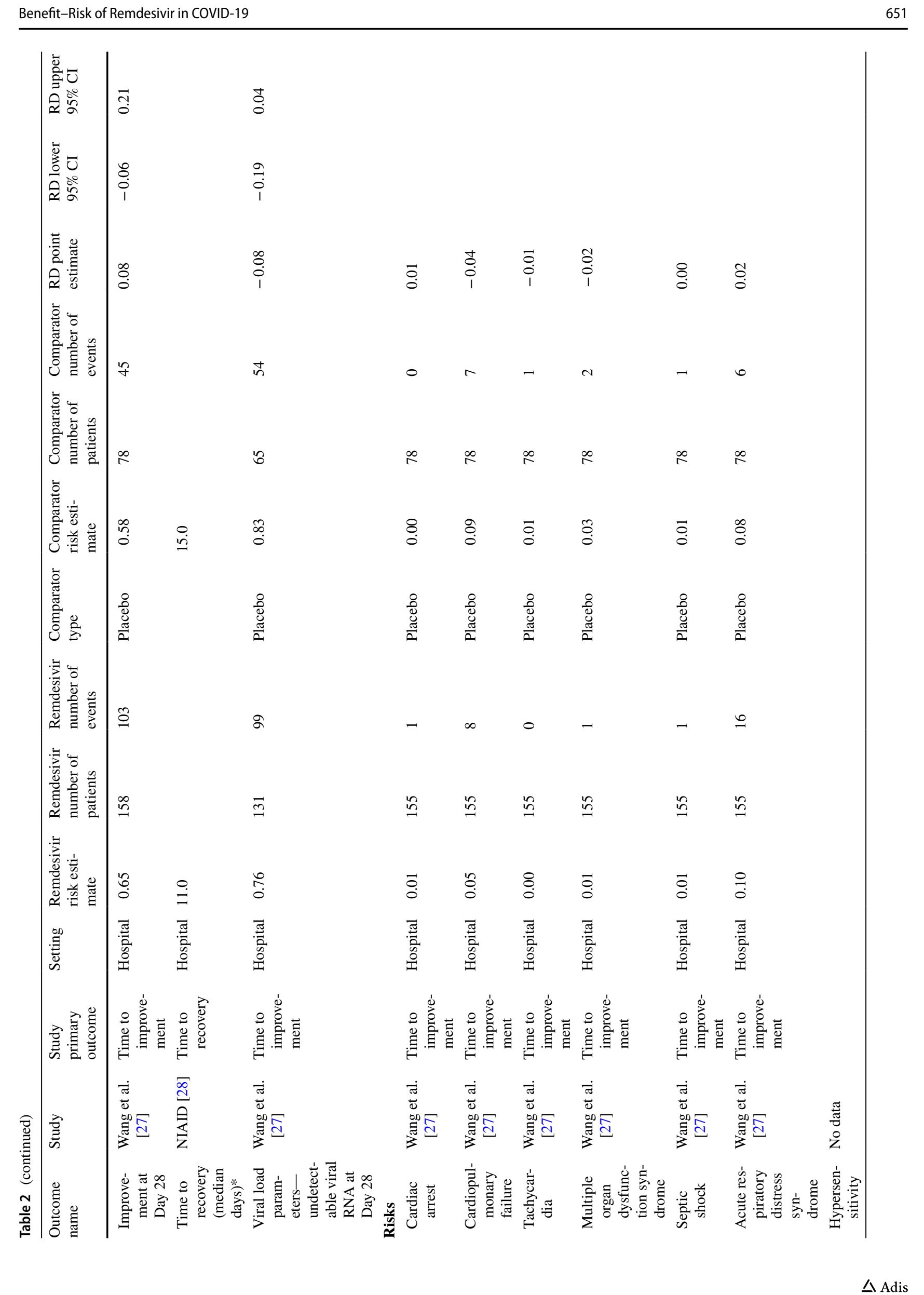




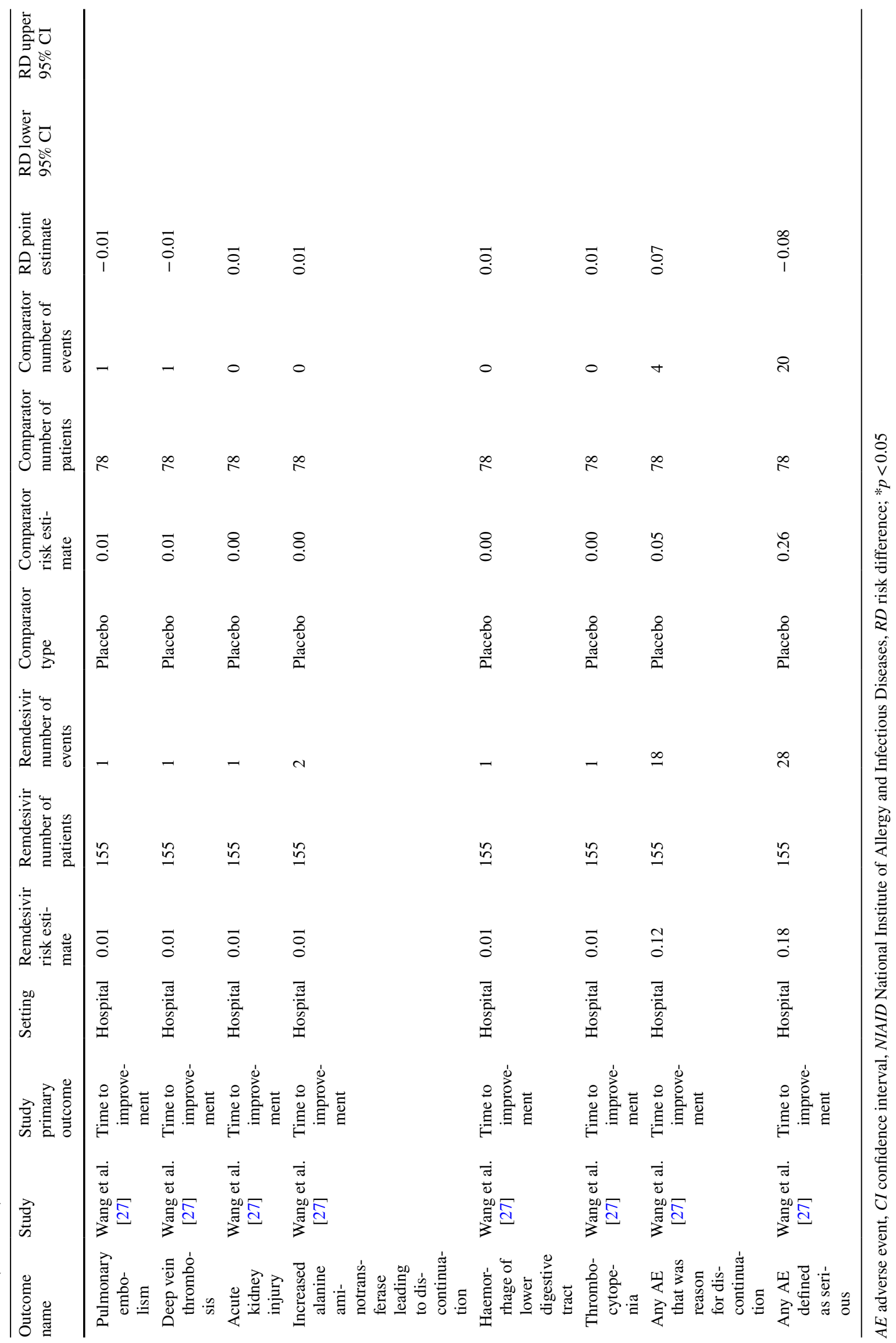


Table 3 Benefit-risk summary table for key benefits and risks identified for remdesivir

\begin{tabular}{|c|c|c|c|c|}
\hline Outcome name & $\begin{array}{l}\text { Remdesivir } \\
\text { risk/1000 } \\
\text { patients }\end{array}$ & $\begin{array}{l}\text { Compara- } \\
\text { tor risk/1000 } \\
\text { patients }\end{array}$ & $\mathrm{RD}(95 \% \mathrm{CI}) / 1000$ patients & Hazard ratio $(95 \% \mathrm{CI})$ \\
\hline \multicolumn{5}{|l|}{ Benefits } \\
\hline Death by Day 29 & 80 & 120 & -40 & \\
\hline Death at Day 28 & 139 & 128 & $11(-81$ to 103$)$ & \\
\hline ICU admission-invasive ventilation at Day 28 & 13 & 39 & -26 & \\
\hline Non-invasive ventilation at Day 28 & 13 & 26 & -13 & \\
\hline Oxygen at Day 28 & 120 & 169 & -49 & \\
\hline Time to clinical improvement (days) & & & & $1.23(0.87-1.75)$ \\
\hline Improvement at Day 28 & 652 & 577 & $75(-57$ to 207$)$ & \\
\hline \multicolumn{5}{|l|}{ Time to recovery (days) } \\
\hline $\begin{array}{l}\text { Viral load parameters-undetectable viral RNA at Day } \\
28\end{array}$ & 755 & 831 & $-76(-192$ to 42$)$ & \\
\hline \multicolumn{5}{|l|}{ Risks } \\
\hline Cardiac arrest & 6 & 0 & 6 & \\
\hline Cardiopulmonary failure & 52 & 90 & -38 & \\
\hline Tachycardia & 0 & 13 & -13 & \\
\hline Multiple organ dysfunction syndrome & 6 & 26 & -20 & \\
\hline Septic shock & 6 & 13 & -7 & \\
\hline Acute respiratory distress syndrome & 103 & 77 & 26 & \\
\hline Pulmonary embolism & 6 & 13 & -7 & \\
\hline Deep vein thrombosis & 6 & 13 & -7 & \\
\hline Acute kidney injury & 6 & 0 & 6 & \\
\hline $\begin{array}{l}\text { Increased alanine aminotransferase leading to discon- } \\
\text { tinuation }\end{array}$ & 13 & 0 & 13 & \\
\hline Haemorrhage of lower digestive tract & 6 & 0 & 6 & \\
\hline Thrombocytopenia & 6 & 0 & 6 & \\
\hline Any AE that was reason for discontinuation & 116 & 51 & 65 & \\
\hline Any AE defined as serious & 181 & 256 & -75 & \\
\hline
\end{tabular}

$A E$ adverse event, $C I$ confidence interval, $R D$ risk difference

a median 15 days (placebo group) to 11 days (remdesivir group) $(p<0.001)$. Further study results provided suggested a non-significant reduced mortality risk amongst remdesivir patients $(8.0 \%)$ versus patients given placebo (11.6\%) $(p=0.059)$, which equates to 40 fewer deaths per 1000 patients treated with remdesivir. A decision was recently made by NIAID to end this trial early because it was deemed to have been unethical to continue given the beneficial effect of remdesivir on the primary endpoint of the study shown at interim analysis [34].

Key risks have been identified and included based on currently available evidence; however, it is acknowledged that the safety profile of remdesivir has not been fully characterized. From risk data currently available, key risks were identified and ranked according to seriousness. The cardiovascular side effects of remdesivir are largely unknown; one patient in the study by Wang et al. was noted to have had a cardiac arrest. Individual cases of multiple organ dysfunction syndrome and septic shock were also reported in this study [27], whilst reports were also identified in patients receiving remdesivir on a compassionate use basis [26]. In the latter case, reports of these events occurred in patients who were on invasive ventilation. Whilst there were multiple reports of liver enzyme abnormalities in patients who received remdesivir in the study by Wang et al. [27], three patients discontinued treatment as a result of liver enzyme elevation (two reports of raised alanine aminotransferase, one report of increased total bilirubin). Reports of acute respiratory distress syndrome have also been reported as adverse events in patients taking remdesivir [27, 31]. Sixteen patients experienced this outcome during treatment in the study by Wang et al. [27], which led to discontinuation in seven patients. Finally, whilst fewer patients experienced an adverse event classified as serious in the remdesivir group 
(18\%) compared with the placebo group (26\%), a higher number of patients discontinued remdesivir as a result of an adverse event (12\% vs 5\%) [27]. The difference in incidence rates observed for adverse events should be interpreted with caution, as causality cannot be inferred and results may have been observed due to chance alone given the underpowered study sample size.

Based on the currently available data, it would appear that remdesivir improves time to recovery and may also reduce mortality compared with patients on placebo, which suggests improved clinical outcomes with remdesivir. Currently, there would appear to be favourable efficacy results from patients treated with remdesivir in the context of severe COVID-19 disease from these preliminary data available. The data in the study by Wang et al. [27] did not include any statistically significant results, although it is acknowledged that the study was underpowered. Primary endpoint data available from the Adaptive COVID-19 trial [28] have also suggested a shorter time to recovery in patients treated with remdesivir, with non-significant reduced mortality risk. There is limited safety data available at the current time, although it is expected that this will increase as more clinical trial safety data becomes available. From the limited clinical trial safety data, it is unclear whether reports of serious adverse events are a function of the underlying severe COVID-19 disease, or attributable to treatment with remdesivir. It is anticipated that both the efficacy and the safety profile will be further strengthened in the coming months with the availability of additional clinical trial data.

\subsection{Strengths and Limitations}

Sample sizes for each outcome were limited to those available in the original studies and may not have adequate power to detect differences in risk between groups, especially where the outcomes examined were not the primary outcome of interest. The benefit-risk assessment is limited by the availability of data, which currently consists of two studies within severe COVID-19. The generalisability of these results to other populations is unknown; for example, application of the Wang et al. results outside of China [27]. However, this assessment can be subsequently updated once further data from clinical trials are available. In addition, given the public health urgency of the COVID-19 pandemic, it is important to provide a systematic assessment of the benefits and risks of remdesivir treatment with evidence available to date and establish a framework that can be used to rapidly update the assessment once further data become available.

Data quality is not reflected in this benefit-risk assessment, though all data was extracted from peer-reviewed manuscripts, with the exception of data included from the Adaptive COVID-19 treatment trial. These data were obtained from the NIAID website (a government agency website), and included time to recovery and mortality data. Safety data have not been published on the website at this time. Since the full results of this clinical trial were not made available at the time of datalock (April 30, 2020), a wNCB analysis was not undertaken because there was a concern that only including efficacy outcomes from the Adaptive COVID-19 treatment trial may bias the wNCB results. It is anticipated that such an analysis can be undertaken in the future when results are made available, to provide further evidence on the benefit-risk profile. Our conclusion is based on a qualitative assessment of the available data and subject to change based on further data availability.

Confirmation of causality was not a requirement for inclusion of data in the BRAT assessment. Patients may have been on other concomitant medications or had other medical conditions at the time of remdesivir treatment. Finally, we considered hospitalisation of patients to reflect severe COVID-19, but we acknowledge that severity of disease may vary regardless of hospitalisation.

\section{Conclusions}

Preliminary clinical trial results suggest that there may be a favourable benefit-risk profile for remdesivir compared with placebo in severe COVID-19 infection and further data on benefits would strengthen this evaluation. There is limited safety data for remdesivir which should be obtained in further studies. The current framework summarises the key anticipated benefits and risks for which further data are needed. Ongoing clinical trial data can be incorporated into the framework when available to provide an updated benefit-risk assessment.

Author Contributions MD assisted with study design, identified outcomes of interest, constructed the value tree, assisted with data extraction, and wrote the first draft of the manuscript. VO assisted with study design, data extraction, writing the manuscript, and wrote the study proposal. SD assisted with study design, identified outcomes of interest and assisted with the construction of the value tree and writing the manuscript. DR, SL and AE assisted with study design, literature searching and writing the manuscript. SAWS assisted with the concept, study design and manuscript revisions. All authors reviewed, contributed to revisions and approved the manuscript and accept full responsibility for its overall content.

\section{Compliance with Ethical Standards}

Funding The Drug Safety Research Unit (DSRU) is an independent academic institution that works in association with the University of Portsmouth. No funding was received for this project.

Conflict of interest The Drug Safety Research Unit is an independent charity (No. 327206) that works in association with the University of Portsmouth. It receives unconditional donations from pharmaceutical 
companies. The companies have no control over the conduct or the publication of the studies conducted by the DSRU. Gilead is providing support for an unrelated methodological project led by the DSRU as part of a large group of pharmaceutical companies, unrelated to remdesivir or any other Gilead product. They were not aware of our decision to undertake this project and provided no financial support or input on the manuscript methods and content. Miranda Davies, Vicki Osborne, Samantha Lane, Debabrata Roy, Sandeep Dhanda, Alison Evans and Saad Shakir have no other conflicts of interest to declare.

Ethics approval This study was conducted in accordance with international ethical guidelines. Ethics approval was not required for this study.

Availability of data and material (data transparency) Data used in this analysis are available from the references supplied.

Open Access This article is licensed under a Creative Commons Attribution-NonCommercial 4.0 International License, which permits any non-commercial use, sharing, adaptation, distribution and reproduction in any medium or format, as long as you give appropriate credit to the original author(s) and the source, provide a link to the Creative Commons licence, and indicate if changes were made. The images or other third party material in this article are included in the article's Creative Commons licence, unless indicated otherwise in a credit line to the material. If material is not included in the article's Creative Commons licence and your intended use is not permitted by statutory regulation or exceeds the permitted use, you will need to obtain permission directly from the copyright holder. To view a copy of this licence, visit http://creativecommons.org/licenses/by-nc/4.0/.

\section{References}

1. Cui J, Li F, Shi ZL. Origin and evolution of pathogenic coronaviruses. Nat Rev Microbiol. 2019;17(3):181-92. https://doi. org/10.1038/s41579-018-0118-9.

2. Wang C, Horby PW, Hayden FG, Gao GF. A novel coronavirus outbreak of global health concern. Lancet. 2020;395(10223):4703. https://doi.org/10.1016/S0140-6736(20)30185-9.

3. WHO. Naming the coronavirus disease (COVID-19) and the virus that causes it; 2020.

4. WHO. WHO announces COVID-19 outbreak a pandemic; 2020.

5. Chen N, Zhou M, Dong X, Qu J, Gong F, Han Y, et al. Epidemiological and clinical characteristics of 99 cases of 2019 novel coronavirus pneumonia in Wuhan, China: a descriptive study. Lancet. 2020;395(10223):507-13. https://doi.org/10.1016/S0140 $-6736(20) 30211-7$.

6. Sanders JM, Monogue ML, Jodlowski TZ, Cutrell JB. Pharmacologic treatments for coronavirus disease 2019 (COVID-19): a review. JAMA. 2020. https://doi.org/10.1001/jama.2020.6019.

7. Gordon CJ, Tchesnokov EP, Woolner E, Perry JK, Feng JY, Porter DP, et al. Remdesivir is a direct-acting antiviral that inhibits RNA-dependent RNA polymerase from severe acute respiratory syndrome coronavirus 2 with high potency. J Biol Chem. 2020. https://doi.org/10.1074/jbc.RA120.013679.

8. Mulangu S, Dodd LE, Davey RT, Tshiani Mbaya O, Proschan M, Mukadi D, et al. A randomized, controlled trial of Ebola virus disease therapeutics. N Engl J Med. 2019;381(24):2293-303. https ://doi.org/10.1056/NEJMoa1910993.

9. Choy KT, Wong AY, Kaewpreedee P, Sia SF, Chen D, Hui KPY, et al. Remdesivir, lopinavir, emetine, and homoharringtonine inhibit SARS-CoV-2 replication in vitro. Antivir Res.
2020;178:104786. https://doi.org/10.1016/j.antiviral.2020.10478 6.

10. Wang M, Cao R, Zhang L, Yang X, Liu J, Xu M, et al. Remdesivir and chloroquine effectively inhibit the recently emerged novel coronavirus (2019-nCoV) in vitro. Cell Res. 2020;30(3):269-71. https://doi.org/10.1038/s41422-020-0282-0.

11. Sheahan TP, Sims AC, Leist SR, Schäfer A, Won J, Brown AJ, et al. Comparative therapeutic efficacy of remdesivir and combination lopinavir, ritonavir, and interferon beta against MERS-CoV. Nat Commun. 2020;11(1):1-14.

12. de Wit E, Feldmann F, Cronin J, Jordan R, Okumura A, Thomas T, et al. Prophylactic and therapeutic remdesivir (GS-5734) treatment in the rhesus macaque model of MERS-CoV infection. Proc Natl Acad Sci USA. 2020;117(12):6771-6. https://doi.org/10.1073/ pnas. 1922083117.

13. Agency EM. Summary on compassionate use: Remdesivir Gilead; 2020.

14. Gilead. Expanded access treatment protocol: Remdesivir (RDV; GS-5734) for the treatment of SARS-CoV2 (CoV) infection (COVID-19); 2020.

15. Gilead. A phase 3 randomized study to evaluate the safety and antiviral activity of Remdesivir (GS-5734 ${ }^{\mathrm{TM}}$ ) in participants with severe COVID-19; 2020.

16. Gilead. Study to evaluate the safety and antiviral activity of Remdesivir (GS-5734TM) in participants with moderate coronavirus disease (COVID-19) compared to standard of care treatment; 2020.

17. Bin Cao P. A phase 3 randomized, double-blind, placebo-controlled multicenter study to evaluate the efficacy and safety of remdesivir in hospitalized adult patients with mild and moderate COVID-19; 2020.

18. Hospital OU. The (Norwegian) NOR solidarity multicenter trial on the efficacy of different anti-viral drugs in SARS-CoV-2 infected patients; 2020.

19. FDA. Coronavirus (COVID-19) Update: FDA issues emergency use authorization for potential COVID-19 treatment; 2020. https ://www.fda.gov/news-events/press-announcements/coronaviru s-covid-19-update-fda-issues-emergency-use-authorization-poten tial-covid-19-treatment. Accessed 18 May 2020.

20. Coplan PM, Noel RA, Levitan BS, Ferguson J, Mussen F. Development of a framework for enhancing the transparency, reproducibility and communication of the benefit-risk balance of medicines. Clin Pharmacol Ther. 2011;89(2):312-5. https://doi.org/10.1038/ clpt.2010.291.

21. PROTECT. BRAT (Benefit-Risk Action Team); 2015. https:// protectbenefitrisk.eu/BRAT.html.

22. Osborne V, Davies M, Roy D, Tescione F, Shakir SAW. Systematic benefit-risk assessment for buprenorphine implant: a semiquantitative method to support risk management. BMJ Evid Based Med. 2020. https://doi.org/10.1136/bmjebm-2019-111295.

23. Nixon R, Dierig C, Mt-Isa S, Stockert I, Tong T, Kuhls S, et al. A case study using the PrOACT-URL and BRAT frameworks for structured benefit risk assessment. Biom J. 2016;58(1):8-27. https ://doi.org/10.1002/bimj.201300248.

24. Holden WL, Juhaeri J, Dai W. Benefit-risk analysis: examples using quantitative methods. Pharmacoepidemiol Drug Saf. 2003;12(8):693-7. https://doi.org/10.1002/pds.794.

25. Sutton AJ, Cooper NJ, Abrams KR, Lambert PC, Jones DR. A Bayesian approach to evaluating net clinical benefit allowed for parameter uncertainty. J Clin Epidemiol. 2005;58(1):26-40. https ://doi.org/10.1016/j.jclinepi.2004.03.015.

26. Grein J, Ohmagari N, Shin D, Diaz G, Asperges E, Castagna A, et al. Compassionate use of remdesivir for patients with severe Covid-19. N Engl J Med. 2020. https://doi.org/10.1056/NEJMo a2007016. 
27. Wang Y, Zhang D, Du G, Du R, Zhao J, Jin Y, et al. Remdesivir in adults with severe COVID-19: a randomised, double-blind, placebo-controlled, multicentre trial. Lancet. 2020. https://doi. org/10.1016/S0140-6736(20)31022-9.

28. National Institute of Allergy and Infectious Disease. NIH clinical trial shows remdesivir accelerates recovery from advanced COVID-19; 2020

29. Long B, Brady WJ, Koyfman A, Gottlieb M. Cardiovascular complications in COVID-19. Am J Emerg Med. 2020. https:// doi.org/10.1016/j.ajem.2020.04.048.

30. Kumar S, Haqqani H, Wynn G, Pathak R, Lipton J, Mahajan R. Position statement on the management of cardiac electrophysiology and cardiac implantable electronic devices in Australia during the COVID-19 pandemic. Heart Lung Circ. 2020. https://doi. org/10.1016/j.hlc.2020.04.001
31. Gilead. Gilead announces results from phase 3 trial of investigational antiviral remdesivir in patients with severe COVID-19; 2020.

32. Boettler T, Newsome PN, Mondelli MU, Maticic M, Cordero E, Cornberg M, et al. Care of patients with liver disease during the COVID-19 pandemic: EASL-ESCMID position paper. JHEP Rep. 2020;2:100113.

33. Jean SS, Lee PI, Hsueh PR. Treatment options for COVID-19: the reality and challenges. J Microbiol Immunol Infect. 2020. https:// doi.org/10.1016/j.jmii.2020.03.034.

34. Herper M. Inside the NIH's controversial decision to stop its big remdesivir study; 2020. https://www.statnews.com/2020/05/11/ inside-the-nihs-controversial-decision-to-stop-its-big-remdesivir -study/. 